\title{
Mobile Probing and Probes
}

\author{
Uffe Duvaa $^{1}$, Rikke $\emptyset_{\text {rngreen }}{ }^{2}$, Anne-Gitte Weinkouff Mathiasen, and Ulla Blomhøj \\ ${ }^{1}$ Learning Specialist, Oticon A/S, Denmark \\ ${ }^{2}$ IT and Designs for Learning, Dep. of Learning \& Philosophy, Aalborg University, Denmark
}

\begin{abstract}
Mobile probing is a method, developed for learning about digital work situations, as an approach to discover new grounds. The method can be used when there is a need to know more about users and their work with certain tasks, but where users at the same time are distributed (in time and space). Mobile probing was inspired by the cultural probe method, and was influenced by qualitative interview and inquiry approaches. The method has been used in two subsequent projects, involving school children (young adults at 15-17 years old) and employees (adults) in a consultancy company. Findings point to mobile probing being a flexible method for uncovering the unknowns, as a way of getting rich data to the analysis and design phases. On the other hand it is difficult to engage users to give in depth explanations, which seem easier in synchronous dialogs (whether online or face2face). The development of the method, its application to the two projects, and the challenges and potentials which were found are discussed in this paper.
\end{abstract}

Keywords: Human Work Interaction Design, Cultural \& mobile probes, online empirical data gathering methods, multimodal methods, IT and designs for learning.

\section{Introduction - Research Area and Focus}

When investigating, it can be difficult for a designer or researcher to ask questions pertaining to a user's work process, task or similar, if the existence of this process or task is not known to the designer/researcher. Also, it is difficult for a user to recall and verbalize all aspects of a process or task, no matter how relevant they may be to convey to the designer/researcher, if the user's attention to issues related to these aspects are not touched upon in the dialog.

One of the authors of this paper had through previous experience from practice (research-based design and research-based consultancy) found herself in this paradox of trying to get to the "heart of things". It seems that applying traditional qualitative methodologies to a digital and online setting does often not bring about much information besides what is being asked about. Moving to more ethnographic approaches as observations and task analysis can be difficult to do online, and requires a lot of hours for both collecting and analyzing data. With methods as online interviews and focus groups, it has been difficult to bring forward the so called non-knowledge. This does not mean that the knowledge is tacit to the users; but simply that when the

P. Campos et al. (Eds.): HWID 2012, IFIP AICT 407, pp. 161-174, 2013.

(C) IFIP International Federation for Information Processing 2013 
questions are not asked; the issues are just not touched upon. This happened using various digital methods, as online interviews, focus groups and textual "describe your workflow" situation.

The cultural probes method is a more explorative approach, which on previous occasions has provided valuable information, in particular when combined with other inquiry- and interview-based methods in traditional face2face situations [12]. Consequently, it became relevant to look for a way to transfer these experiences to digital and mobile methods.

Based on the before mentioned difficulties with getting to non-knowledge, the first research question was to see if mobile probes can provide an adequate way forward. A second research question was to investigate, which adaptations of the method were useful when gathering qualitative empirical data from end-users, to be applied to the design process.

Two projects have been involved in this research so far. In 2010 the method was used in a project on mobile learning, where school children from the same school in 8.grade participated (young adults at 15-17 years old). Here, the objective was to gain knowledge from users, in order to inform the design process of a mobile application to be used in the educational system. The other and most recent use of the method was in 2011, where the participants were employees from a major Danish consultancy company. This project had a dual objective: One perspective was to learn about the employees' standpoint (feelings about) and use of the existing knowledge sharing platform, in relation to how they perceived the concept of knowledge sharing, and their knowledge sharing strategies in practice. The project also wanted to discover and map possibilities for mobile support of knowledge sharing in the company.

A common focus area, on the practical level of these two involved projects, has been the need to acquire knowledge directly from the end-users; on their work tasks and processes related to knowledge sharing in general and on their use of digital media, more specifically mobile phones / smart phones.

The tasks under investigation were also carried out in a very mobile or flexible environment. For example the employees in the consultancy case, worked from different locations, and only sometimes at the office. Similarly, the young adults were in and out of school, at home, with friends and so forth. Both projects wanted information about a specific group of people (contextual information) and their current use of their smart phones in general and in relation to how future use-situations could be. In the situation of the consultancy the employees were even situated in different countries, making the need for a digital empirical gathering tool, even more eminent.

Research findings from these two projects points to: applying mobile probes in an almost qualitative interview fashion, utilising the users own mobile equipment can be rewarding for the project in term of uncovering non-knowledge; and that making use of multimodal approaches in the data creation, gathering and analysis phase assist sense-making processes. However, in some situations the mobile probes are seen as too intrusive to daily practice (understood as too time consuming for users to deal with).

In the following, a brief presentation of the concept of non-knowledge and a discussion of its implications for this paper is given. Afterwards, a presentation and 
discussion is given of the literature and history on cultural probes as it relates to the development of this mobile probing method. Then the first research design considerations are outlined in the first use of the method. ${ }^{1}$ Afterwards the second project and its methodological use is presented, followed by a general discussion on findings related to the mobile probing method.

\section{Non-knowledge}

Non-knowledge is the knowledge that depends on context, social relations and artifacts in order to become understood or recognized as significant and to be codified.

An integral aspect of the mobile probe method is its ability to help gather otherwise hard to handle knowledge. This paper does however not offer space nor is it the place for a long discussion on the differences between tacit and explicit knowledge, on codified and uncodified knowledge and on focal and subsidiarily knowing, to put it in Michael Polanyis terms, and the often used quote that "There are things that we know but cannot tell. " [16] We would however, like to establish this frame: the knowledge form, which is addressed here, is often both contextually, socially and technologically tied, which means it can be very hard, even impossible, for a participant to describe; as work processes and tasks of everyday life is tied to complex network of actions and interactions, thus it can be difficult to realize the causalities; which relations makes which difference. Non-knowledge however, can be, but is not necessarily tacit knowledge.

If space permitted, it would make sense to also discuss and refer to Ed Hutchins distributed cognition [17], as well as the larger body of knowledge sharing literature, for example Nonaka and Hirotake [18], as the concept of non-knowledge used in this paper, is closely related to the codifiability of interpersonal knowledge.

For the work presented in this paper, the postulate is that a part of the code is kept outside the person "holding" the studied knowledge. This means that this "outside", whether artifact or human, needs to be documented or brought into play, if a larger piece of the sum-knowledge (which is not an a priori definite sum) is to be shared or understood in design and design-research. This makes it necessary to identify relational ties between the studied knowledge and the context and artifact that it is interwoven with. Without gathering this data, the state of the knowledge is in danger of getting perceived as everything is tacit knowledge, which cannot be made explicit, while in fact some is pre-codified and can be "talked about", when connected to the "outside" documented nodes.

With a somewhat different objective, though not far from, Lotte Dars $\varnothing$ in one of her earlier papers with the title: Is there a formula for innovation? Defines and describes non-knowledge as part of her model for innovation. "Non-knowledge deals with that which we know, we do not know; that which we do not know, we do not know; and finally that which we did not have a clue about, that one could know. The problem with non-knowledge is, that is it an uncertain and fear-provoking area to be

1 Research on the first project, with a focus on the domain findings: designing for mobile learning, is published in [11]. 
in, and this leads to, that most people prefer to withdraw to safer grounds.. [and she continues, when referring to how we can work with non-knowledge] .... This is primarily done by asking questions - and keep asking questions." (translation from [19] p.6. The term non-knowledge as used by Dars $\emptyset$ has been developed in collaboration with her then ph.d. advisor Henrik Herlau).

An integral aspect of this view is that the design-researcher, who is in both an analytical and design activity of getting to know the users, context and technologies, and who is mindful to documentation in-situ; also need to ask question to oneself and the process: what has not yet been written, talked about, taken pictures off; which relations did I not see; what can surprise me; and what can I learn now, that I could not predict before?

\section{Methodological Considerations}

The Cultural Probes method was originally introduced to HCI by Gaver, Dunne and Pacenti. The method was developed using probes such as maps, postcards and other materials that were designed to provoke inspirational responses from elderly people in diverse communities [4]. The method provided empirical user generated data, whose content could not be predicted and which held valuable information about emotions, reasons as well as visual representations. This data collecting process preceded the design phase and contributed to the qualitative knowledge-base about users. [4,5].

The typical cultural probing process has used a large degree of openness and anonymity. For example the postcard could contain a statement or question, as open as "what is a living room to you", and some would return with a description of their own living room, others with a dream scenario, some focus on the layout, others on activities etc. As the post card would be pre-stamped or handed in to a box, the anonymity would be well preserved.

In the presented projects in this paper, there was a need to know something about the tasks and processes from a known and pre-defined user group, within a relatively well defined area. Anthropology, where the researcher/designer is present over longer periods of time is suitable, when investigating tasks and processes, also in our situation, where digital and mobile supported tasks and processes needed be investigated (see for example [20]). Full scale methods of "going native", being with the users (traditionally and digitally) at any time over longer periods, are often too resource consuming for many smaller research and development projects. This primarily concerns two aspects: time used to plan, conduct and analyze the data gathered, and time required from the participants. The latter was especially of concern in the consultancy case and would be in many similar situations. The anthropologist way, also lack the voices of the users'. Interviews and focus groups would be feasible, but could not stand alone, as the openness to the direction and type of data received from users as in the cultural probes was also desired.

A hybrid probing method was thus selected based on both appropriateness and scale of method according to the project size. Others have used hybrid probing methods, as using them in a game-environment, which in the cases listed significantly 
increased the number of responses [13], and the cultural probes have also moved to digital probing materials and in recent years in particular to focus on mobile probes using mobile phones [1]. Iversen \& Nielsen applied mobile phones, as they needed to utilise a media which was suitable for children [7]. In a Finnish study by Hulkko et al, the aim was to make the probe more accessible in all contexts. Their research was rooted in a customer survey perspective, investigating the buying habits and decisions people make when buying clothes (thoughts and visual impressions). Consequently, they too digitized the cultural probes concept, handing out mobiles with attached cameras, with the intention of getting empirical data gathered in-situ [6]. The review of mobile probes studies also indicates that the method is predominantly used in the consultancy domain as opposed to the research domain where it focused more on input to practice. For example, one company developed a mobile based 'probe pack'. A tool for collecting data, with the purpose of reducing development and execution time associated with the use of probes [10].

Two arguments are used when applying the digital probe formats: it provides means for getting access to situated information, and users today recognize and are motivated by mobile phones in the same way as disposable cameras and post cards was [7].

The digital based probes are sometimes linked to Experience Sampling, used in a form of non-present observation of the everyday life of respondents. Experience sampling was originally described as: "to identify and analyze how patterns in people's subjective experience relate to the wider conditions of their lives. According to the authors, the purpose of using this method is to be as "objective" about subjective phenomena as possible without compromising the essential personal meaning of experience" [14]. However, in our context we argue, that technology focused data gathering, using handed-out technology will not provide a valid technology usage picture. Instead it will most likely give insight into the adaptation and acquisition of new technology. While the Experience sampling method seeks to minimize this by prompting for in-situ, descriptive data; But if mobile phones are handed out as part of the empirical data gathering process and the data gathering process is about mobile phones: how natural would their use be to their everyday?

That is, in the above studies the participants were given new tools (hard-/software), which they had to learn to use, before being able to respond on the probe tasks. If such a strategy is applied, it may seem extensive and intrusive to the participants, because learning a new media and being able to use it actively, is a process that takes time and energy. Even if the learning curve over time becomes shorter for some user groups (that is the time needed for acquiring the ability to use a new smartphone, may be relatively small for some user groups), it is not part of a natural work situation to carry several phones; to work on one phone while carrying out the usual digital tasks and then shift to a new phone to send data to the research project.

Furthermore, in the projects, the mobile phone was not only acting as an agent for acquiring empirical data (as the situation in hitherto mobile probe settings as seen above), the mobile was also the technological medium for which data should say something about (mobile learning and knowledge sharing / management). Accordingly, not only the content of the data but also the ways in which data was delivered and 
handled, provided a valuable dimension for investigating mobile use. It would not make sense to give the participants a new phone, with different operating systems and apps than they usually use, as it would say less about their everyday informal strategies.

Probes have often been applied in a manner, where participating people was probed randomly, with pre-designed probes and answer-channel (i.e. a question-probe + take picture that answers question, or a theme-probe + write a text about the theme, etc.) or by allowing participants to choose time and answer-channel $[4,5,6]$. There are no immediate follow up on the returning data from the participants' [1]. Boehner et al. argue for probes that work as an open conversation with the participants. A conversation, where the researchers' contribution is a response to what was expressed in the answers coming in from the participants', instead of making representations that delimit the design space [1]. As a result, a more dialog oriented approach, seem appropriate [2].

In a semi-structured interview, the interview guide is pre-formulated [8]. The guide sets the scene, but enables the interviewer to come with follow-up questions, to guide the participants in new directions or to even omit subjects from the pre-formulated guide if this is relevant in the context as the interview takes place.

An important aspect of the cultural probes method is that it does not aim at generating data, which should go through a traditional qualitative analysis; rather the objective is to make the researcher sensitive towards the emotions, experiences and causalities of the participating group [4]. The initial focus of the probes has thus newer been on the development of products, but on new understandings of users and their use of technology [5]. However, in the research and development projects outlined in this paper, the objective has been to understand users' application and utilization of existing technology in order to make future design decisions or strategies of design. This may influence the original method in terms of how the received data becomes sorted and analyzed. In "Moving from cultural probes to agent-oriented requirements engineering "Anne Boettcher discuss data collection in social environments via cultural probes and uses socially oriented requirements analysis for informed technology production [15]. However, mobile proping in these settings does not take place in a vacuum, but in projects were a number of other design activities take place. The intention would not be to let the responses to the probes be subjected to a direct translation into a design, or to see them as "correct answers to a problem". The idea is rather to support the codification and understanding of all data from all methods; to rest on a better foundation of users, their opinions and emotions, and their everyday practice.

In summary and for the outlined contexts, the combination of methods could make it possible to expand on the dialogical aspect, as the mobile phone enables a more flexible probing process than pre-printed post cards and similar material, using generic programs familiar to the participants (as sms, mms, mail etc.). Also, where interviews and surveys have a more retrospective character and ethnographic or anthropological research often requires a "researcher to go native"; in this kind of mobile probing the researcher could ask to the "here and now" of a situation and alongside the actual answers, receive valuable multimodal metadata pertaining to contextual 
technology usage. The probes are part of a larger set of methods applied in pre-phases and data from the probes are analyzed and joined with data from the other methods.

Consequently, for these studies the Mobile Probes uses a semi structured interview approach, combined with non-intrusive technology, namely their everyday tool, their own mobiles.

\section{First Use - Mobile Learning in Schools}

Through ministerial funds a project partnership was formed between a broadcasting corporation and two private companies with the objective to develop a learning application for smartphones. The vision was to combine digital information from archives with current information, and thereby support learning in multiple disciplines (as relating historical and current news shown on the phone as the user moves in the city spaces). One of the authors of this paper was invited as a research-based consultant within IT didactical design and two other authors participated as research assistants, where they applied the data in their master studies. IT didactical design refers to the theories, models and practices of teaching and learning supported by IT, focusing on the process perspectives in educational design, as activities relating to both (re)designing, planning, running, adapting and evaluating learning processes and spaces for learning.

The pre-phase began in the spring of 2010. However, though the researchers finalised the pre-phase, the partnership between the companies dissolved and the project itself was newer finalised. Nevertheless, the process and findings were from a research perspective methodological relevant.

The project's primary audience was students at $15-17 \mathrm{y}$. According to the project description: in 2009 more than $98 \%$ of these Danish students were using mobile phones, one third send photos, videos or similar and roughly one in five surf the web using their mobile phones. However, in 2010 very little was known about the informal strategies, the cognitive and subjective processes, as well as the reasons and motivations for mobile applications use. A small investigation based on the Cultural and Mobile Probe method was seen as an adequate way forward at the same time as workshops and low-fi prototypes were designed and evaluated. The research design was build on the assumption that the probe data would spread light on what are the positive and negative user experience elements, and how these components influence the students' choice and learning strategies.

Access to the potential participants, was gained through the headmaster in order to get permission to contact them during school hours. All students in the age group were presented to the project and encouraged to contact the researcher if they wished to participate. The final participants were chosen from those who replied, based on criteria's related to their access to the technology, and their experience with smartphones. Subsequently, four students from the same high school were chosen, adopting a homogenous and typical sampling based strategy [2].

The probing took place over a period of seven days in which the participants received text messages with various questions about their use of smartphones, and 
received tasks they should address utilising various media and generic smartphone functions. The participants received three text messages each day. The first question was typically sent in the morning, the second at noon, and the third around 6 o'clock in the evening. The first two questions were almost always the same for all participants, whereas the third was often used as a follow-up question to their previous responses, making it individual. Three questions per day enabled some of the positive aspects of semi-structured interviews, where you can make inquiries in to the essence of opinions [8].

During the seven days, 81 tasks were sent and 74 answers were received. The answers were primarily text messages, but also many pictures / screen shots. (table 1 and figure 1).

Table 1. Number and type of responses

\begin{tabular}{|c|c|c|c|}
\hline \multicolumn{3}{|c|}{ Number of tasks send: } & 81 \\
\hline \multicolumn{3}{|c|}{ Number of responses recieved: } & 74 \\
\hline \multicolumn{4}{|c|}{ Media used in responses*; } \\
\hline SMS: & 57 & \multirow{3}{*}{\multicolumn{2}{|c|}{$\begin{array}{l}\text { *Some answers had both } \\
\text { text and pictures }\end{array}$}} \\
\hline \multirow{2}{*}{\begin{tabular}{|l} 
Audio: \\
Pictures.
\end{tabular}} & 4 & & \\
\hline & Pictures: & & \\
\hline
\end{tabular}

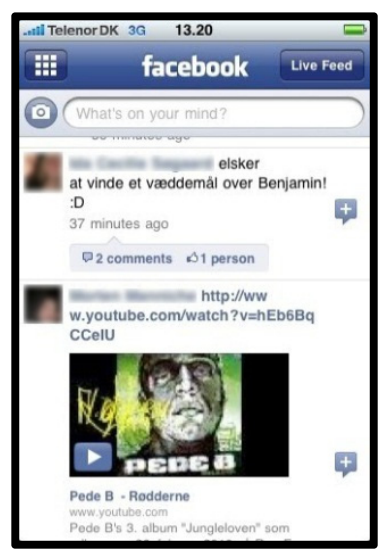

Fig. 1. Example of probe response picture

From an analytical perspective, the Mobile probe method objective was not to reach a single unambiguous answer or solution, but rather the responses should let the researcher become inspired by the participants [1]. With the aim to support the project's rigidity in the analysis phase, while keeping a user-centric focus, user experience goals and cognitive process variables were applied to the analysis. The cognitive processes were identified as: attention, perception \& recognition, memory, learning and problem-solving, planning etc. The user experience goals were identified, as: satisfying, enjoyable, provocative, boring, etc. (All derived from [9]).

The multimodal nature of the responses presented a number of challenges during both the collection and analysis process. The primary challenge was to organize the data in a way that would give it a uniform structure and expression, in order to compare answers and reveal trends and relations, to identify and codify the nonknowledge. Data also had to be recorded and stored during the collection process, in a way that supported the interview-based probes approach. Consequently, the project identified "probe managers", sending tasks to the participants and communicating with them when necessary. To facilitate this, an online data collection site was used, 
where answers and thoughts on the ongoing process was shared. This site allowed simultaneous work on the data and a kind of reflection-log.

For example a simple text-based table was used to store and organize both the predefined guide and questions, as well as the participants' answers; Day 1, Question A, participant 1, 2, 3, 4 - Question B, participant 1, 2, 3, 4, etc. Organizing data this way enabled an ongoing preliminary exploratory analysis during the collection phase [2]. This overview helped qualify the follow-up questions, and showed if it was necessary to deviate from the original questions. After the probing process had ended, a thematic analysis based on predetermined themes began [2]. The theme-set used was based on the reflective Cognitive Processes (CP) found in the participants smartphone use and the Subjective User Experiences (SUE) associated with the actual use [9]. Through physical and visual (not online) ordering of the relations in the data, it was possible to study and discuss which cognitive categories and subjective user experiences were dominant in the data, and gain insight into the correlations between these and different forms of smartphone interaction (figure 3 ).

\begin{tabular}{|l|}
\hline \multicolumn{2}{|c|}{$\mathbf{3 A 3}$} \\
\hline Answer category: explain favorit app \\
\hline Summary: Facebook is favorit app \\
most used, good design and user-friendly \\
\hline SUE: enhancing sociability \\
emotionally fulfilling, enjoyable \\
\hline $\begin{array}{l}\text { CP: Attention } \\
\text { perception and reconition }\end{array}$ \\
\hline
\end{tabular}

Fig. 2. Each piece of user material was first analyzed

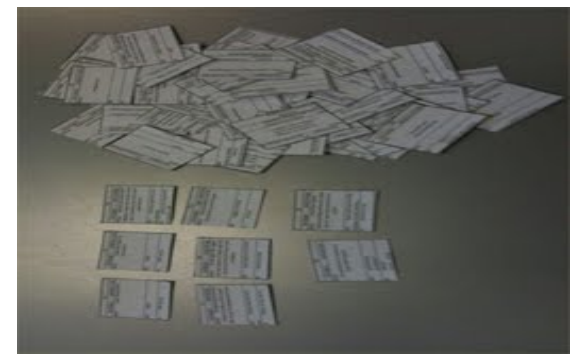

Fig. 3. Then all pieces were analyzed as a collective

Example: Dohn and Johnson describe m-learning strategies and the use of the students' own smartphones as an advantage. When the students already know the media, they are already competent and can rapidly decode relevant strategies and instead focus on the learning content. They found students use small one-player games, and they suggest including these in formal learning applications [3]. But what happens when one adopts the cultures and behaviors' of the informal strategies into a formal learning setting? This may be valuable, but can also be disadvantageous in some situations. As such, we found in this project's mobile probe data also showed use of small one-player games. The probe process, however, disclosed that they were played as a fast game "to kill time", or while waiting for something else to happen (during transport etc). If designed into learning software, the culture of "beating the game" fast, rather than learning about the domain knowledge, may be inherited. 


\section{Second Use - Knowledge Sharing in Consultancy}

This project was conducted in 2011 and it is noteworthy that the consultancy company had not asked in advance for the research to take place, but decided to use a great deal of effort and man-hours, once they were introduced to the ideas (of the whole pre-phase). The company was in the midst of reorganizing their knowledge sharing platform and had discussed the possibility for mobile support, but had not yet investigated this nor made any decisions regarding this. The total pre-phase was part of the master students' thesis, and the primary research focus was the mobile probing method and how it worked in a pre-phase.

Prior to the mobile probing process, the potential participants received an introduction to the method and to the investigation at hand. It was the employees nearest manager who decided, who participated. It was decided to keep the qualitative approach, and only 6 people participated. There were 9000 employed in the company globaly, making it a truly small, but still interesting insight in to the everyday practices and feelings towards knowledge management in the specific work-domain. For the mobile probing process a mobile probe guideline was developed, similar to the idea of an interview guide. The objective was to understand more about these employees everyday knowledge sharing practice and to learn about the terms and phrases they themselves used for processes related to knowledge sharing. The two tables below illustrate this point and summarize the number of mobile probes and responses sent. Responses were very different in nature, sometimes very concrete ways of working (as in table 3), other times with suggestions to design or just thoughts.

Table 2. Summary

\begin{tabular}{|c|c|c|c|}
\hline \multicolumn{3}{|c|}{ Number of tasks send } & 90 \\
\hline \multicolumn{4}{|c|}{ Number of responses recieved } \\
\hline \multicolumn{4}{|c|}{ Media used in responses*: } \\
\hline SMS & 78 & \multirow{2}{*}{\multicolumn{2}{|c|}{$\begin{array}{l}\text { "Some answers used } \\
\text { more than one mediatype }\end{array}$}} \\
\hline Mail text & 14 & & \\
\hline Picture & 6 & & \\
\hline Audio & $\underline{2}$ & & \\
\hline Video & 1 & & \\
\hline
\end{tabular}


Table 3. Example of question and answer

\begin{tabular}{|c|c|}
\hline \multicolumn{2}{|c|}{$4 \mathrm{E} 3$} \\
\hline \multicolumn{2}{|c|}{$\begin{array}{l}\text { Hi XXX. Time for thursdays last task and the last message you will get from me. } \\
\text { Please tell me about a situation in which, input from a colleague changed your } \\
\text { working practice. I look forward to getting your responses to thursdays tasks. } \\
\text { /XXX }\end{array}$} \\
\hline \multicolumn{2}{|c|}{$\begin{array}{l}\text { It was highlighted to me by a colleague that getting caught up in the old } \\
\text { emailknowledge share medium was not only clogging up my mailbox but wasn' } \\
\text { efficient. By using SharePoint to upload items, using announcements and then } \\
\text { simply sending a link in an email should become the norm for everyone. This is } \\
\text { now the way I communicate on the UK. }\end{array}$} \\
\hline Sent & Recieved \\
\hline Media:SMS & Media:SMS \\
\hline \multicolumn{2}{|c|}{$\begin{array}{c}\text { Tech options - social practice - know how - internal knowledgesharing - } \\
\text { tech effiency }\end{array}$} \\
\hline
\end{tabular}

In retrospect, when analyzing the time of probe distribution, it becomes obvious that the probes consistently was distributed at 9:00, 12:00 and 16:00. Though not an explicit decision, clearly the research team shared a notion on when a probe may be more or less intrusive. Already at day 1, one of the respondent expressed that the probing was a stress factor, responding with a "I am busy these days, so please make it brief". This adds a layer to the mobile probing process as it took place in this context, where the respondent was more or less chosen by the nearest managers and thus not volunteered. (Which also became part of the discussion in the analysis and interpretation phases.)

To use this insight in the pre-phase project results, the analysis took point of departure in a set of pre-defined themes, derived from the existing data (meeting, materials and observations from the consultancy company). A possible drawback here was an increased risk for contamination of the data analysis, by inducing ones preconceptions as they are or have been "build" in the analysis of the other data. The incremental, iterative analysis process, where the raw data is revisited and where the themes are discussed after each cycle (leading to reformulation or even to a theme being discarded), aims at minimize this risk.

Mobile probing is a method that by explicit choice is digital. Nevertheless the analysis process makes use of a physical representation and handling / re-coding process; a process that takes place after all mobile probes are collected. Good network views are available in digital analysis tools as Atlas.ti, but the bodily engagement and collaborative dialog while doing so was prioritized here. Soon, technologies may support the analyst in an acquiring equally sense of the data, but for now this was a very adequate process. 


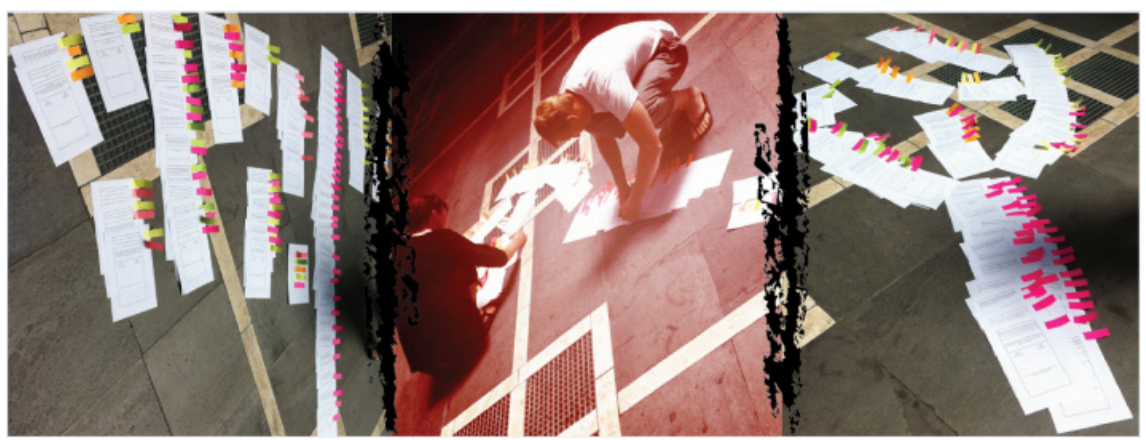

Fig. 4. Bodily engagement into the collaborative analysis

\section{Discussion of Experiences and Conclusions}

As every respondent participate in parallel at a given time period, the method provides a setting for getting access to people independent of space and time. The probing aspect gives knowledge about what the person is doing, but at the same time makes it possible for the researchers or practitioners to make follow-ups based on previous responses. Questions that arose were: What does this mean? Which led to an individual follow-up question; Are other people in similar situations? This led to a follow-up question to all participants. This mean the Mobile Probing method provide the possibility to work with gaining some continuity by asking every participant the same question in new ways, or new questions all together. This is different from traditional interviews, where the basis of the guide may change over time (as interviews are carried out), though follow-up questions of course can and do take place, it means starting up the relation again. Here, the continuity and changes can happen to every participant during the mobile probe period.

However, it was often difficult to receive in-depth answers. This is likely to be linked to two reasons: primarily willingness to invest time to answer the researcher/designer as seen in relation to this time could be used on tasks related to ongoing work requirements. Secondly, based on choice of medium (though not directly investigated in these two projects), in particular SMS, but also when answering mails on a phone, makes answers somewhat short. There is the same tendency in chatinterviews.

The mobile probing process as used here, made use of a large analytical phase in order to use the incoming data in the design phase, whether for design of products or design of processes. The physical sorting of data, provides the team with a very dynamic and visual representation, but at the same time this process needs logging with pictures or similar, to be preserved. And similar a cloud-based storage solution for the mobile probe materials is essential in making it possible to gain access to all the material regardless of time and location. This allowed for everyone in the project team to get an overview of all the responses, as they are received, and thereby ensured the qualification of the open and ongoing dialog. 
In conclusion, the mobile probing method and the mobile probes used, differ from the existing and original cultural probes in that: During the information gathering process the mobile probes takes use of a qualitative interview-based method, with focus on an authentic dialog. This gives the researchers the possibility to get to know the researchers and the participants the researchers. Through this, the probing method can allow for facilitating the participants, pursue interesting points etc.

Secondly, the mobile probing process used a combined analysis strategy, with predefined themes, allowing the on-going analysis of probes as they were received and then later in a physical coding process as well. Also, making use of multimodal approaches in the data creation, gathering and analysis phase assisted the sense-making processes.

Using mobile probes can be a very rewarding for the projects in term of uncovering non-knowledge, but in some situations the mobile probes are seen as intrusive (understood as too time consuming for user to deal with).

\section{References}

1. Boehner, K., Vertesi, J., Sengers, P., Dourish, P.: How HCI Interprets the Probes. In: Proc. of CHI 2007, San Jose, CA, USA (2007)

2. Cresswell, J.W.: Educational Research - Planning, conducting, and evaluating quantitative and qualitative research, 3rd edn. Pearson, USA (2008)

3. Dohn, N.B., Johnsen, L.: E-læring på web 2.0. Samfundslitteratur, Frederiksberg (2009)

4. Gaver, D., Pacenti, E.: Design: Cultural probes. Interactions 6(1), 21-29 (1999)

5. Gaver, B., Penningtong, W.: Cultural probes and the value of uncertainty. Interactions 11(5), 53-56 (2004)

6. Hulkko, S., Mattelmäki, T., Virtanen, K., Keionen, T.: Mobile probes. In: Proc. of the 3rd NordiChi, vol. 82, pp. 43-51. ACM (2004)

7. Iversen, O.S., Nielsen, C.: Using Digital Cultural Probes in Design with Children. In: Proc. of IDC 2003, p. 1 (2003)

8. Kvale, S.: InterView - En introduktion til det kvalitative forskningsinterview. Hans Reitzels, Copenhagen (1997)

9. Preece, J., Rogers, Y., Sharp, H.: Interaction design, Beyond human-computer interaction, 2nd edn. John wiley \& sons, Ltd. (2007)

10. Mobile Probes: Frontpage and product descriptions on (2010), http: / / www . Mobi leprobes . dk (visited June 25, 2010)

11. Blomhøj, U., Duvaa, U., Ørngreen, R.: Mobile Probes in Mobile Learning, pp. 1-4. Institut for Uddannelse og Pædagogik, Aarhus Universitet, København (2012)

12. Ørngreen, R., Andreasen, L.B., Levinsen, K.: Digital storytelling: the methodology applied and example stories from Denmark, København (2010)

13. Bernhaupt, R., Weiss, A., Obrist, M., Tscheligi, M.: Playful Probing: Making Probing more Fun. In: Baranauskas, C., Abascal, J., Barbosa, S.D.J. (eds.) INTERACT 2007. LNCS, vol. 4662, pp. 606-619. Springer, Heidelberg (2007)

14. Csikszentmihalyi, M., Larson, R.: The Experience Sampling Method. New Directions for Methodology of Social \& Behavioral Science 15, 41-56 (1983)

15. Boettcher, A.: Moving from cultural probes to agent-oriented requirements engineering. In: OZCHI 2006 Proceedings of the 18th Australia Conference on Computer-Human Interaction: Design: Activities, Artefacts and Environments, pp. 253-260 (2006) 
16. Polanyi, M.: Tacit Knowing: Its Bearing on Some Problems of Philosophy. Reviews of Modern Physics 34(4), 601-616 (1962, 1996)

17. Hutchins, E.: Cognition in the Wild. MIT Press (1995)

18. Nonaka, I., Hirotake, T.: The knowledge creating company (1995)

19. Dars $\varnothing$, L.: Findes der en formel for innovation? Børsens Ledelseshåndbøger, 12 sider (2003)

20. http://digitalanthropologist.blogspot.com/2009/11/digitalanthropology-vs-digital.html ((re)accessed February 18, 2012) 